\title{
A Fractional-Order System with Coexisting Chaotic Attractors and Control Chaos via a Single State Variable Linear Controller
}

\author{
Ping Zhou ${ }^{1 D},{ }^{1,2}$ Meihua Ke, ${ }^{2}$ and Peng $\mathrm{Zhu}^{2}$ \\ ${ }^{1}$ Center of System Theory and Its Applications, Chongqing University of Posts and Telecommunications, Chongqing 400065, China \\ ${ }^{2}$ Key Laboratory of Network Control and Intelligent Instrument of Ministry of Education, Chongqing University of Posts and \\ Telecommunications, Chongqing 400065, China
}

Correspondence should be addressed to Ping Zhou; zhouping@cqupt.edu.cn

Received 23 March 2018; Accepted 16 April 2018; Published 25 June 2018

Academic Editor: Viet-Thanh Pham

Copyright (C) 2018 Ping Zhou et al. This is an open access article distributed under the Creative Commons Attribution License, which permits unrestricted use, distribution, and reproduction in any medium, provided the original work is properly cited.

\begin{abstract}
A 3D fractional-order nonlinear system with coexisting chaotic attractors is proposed in this paper. The necessary condition of the existence chaos is $q \geq 0.8477$. The fractional-order system exhibits chaotic attractors with the order as low as 2.5431 . The largest Lyapunov exponent varying as fractional order $q$ is given. Furthermore, there are the coexisting "positive attractor" and "negative attractor" in this fractional-order chaotic system, and the necessary condition for "positive attractor" and "negative attractor" is obtained. Meanwhile, a control scheme for the stabilization of the unstable equilibrium is suggested via a single state variable linear controller. Numerical results show that the control scheme is valid.
\end{abstract}

\section{Introduction}

Chaotic behaviors in nonlinear is a very interesting phenomenon. The high irregularity, unpredictability, and complexity in chaotic systems $[1,2]$ have been widely used in the field of engineering and technology such as secure communications, image steganography, authenticated encryption, motor control, and power system protection. Recently, coexisting chaotic attractors have been found in chaotic systems [3-9]. For example, the coexisting chaotic attractors in a 3D no-equilibrium system were reported by Pham et al. [3], the coexisting multiple attractors in Hopfield neural network were found by Bao et al. [4], the coexisting chaotic attractors in a hyperchaotic hyperjerk system were given by Wang et al. [5], the coexisting "positive attractor" and "negative attractor" in a $3 \mathrm{D}$ autonomous continuous chaotic system were found by Zhou and Ke [6], and so on [7-9]. Therefore, more and more attention has been focused on the coexisting chaotic attractors in nonlinear chaotic systems.

On the other hand, the fractional-order differential equations [10-12] can be accurately described in the real-world physical systems such as viscoelasticity, dielectric polarization, electrode-electrolyte polarization, electromagnetic waves, heat conduction, diffusion-wave, and superdiffusion. Chaotic behaviors have been found in many real-world physical fractional-order nonlinear systems, for example, the fractional-order chaotic brushless DC motor [13], the fractional-order electronic circuits [14], the fractional-order microelectromechanical system [15], and the fractional-order gyroscopes [16]. Therefore, more and more attention has been paid to the chaotic behaviors in fractional-order nonlinear systems.

Motivated by the above discussions, based on a 3D autonomous continuous chaotic system reported by Zhou and $\mathrm{Ke}$ [6], we suggested a 3D autonomous continuous fractional-order system. We have shown that the chaotic system reported by Zhou and Ke [6] can be extended to its fractional-order version where the coexisting "positive attractor" and "negative attractor" can be observed. We obtained that the fractional-order system with the order as low as 2.5431 exhibits chaotic attractors. Moreover, we obtained the largest Lyapunov exponent varying as fractional order. Finally, for the stabilization of the unstable equilibrium, one control scheme is proposed via a single state variable linear controller. 
The outline of this paper is organized as follows. In Section 2, based on a 3D autonomous continuous chaotic system reported by Zhou and Ke [6], the fractional-order version nonlinear system is given, and some basic dynamical properties of this fractional-order version nonlinear system are obtained including the necessary condition of the existence chaos, the largest Lyapunov exponent varying as fractional order, and the coexisting "positive attractor" and "negative attractor." In Section 3, by a single state variable, stabilization of the unstable equilibrium points of the fractional-order chaotic system is discussed. Finally, the conclusions are given in Section 4.

\section{System Model and Basic Characteristics}

In this paper, the Caputo definition of the fractional derivative will be used in next. The Caputo definition of the fractional derivative is described as

$$
\begin{array}{r}
{ }_{0}^{c} D_{t}^{q} g(t)=\frac{1}{\Gamma(k-q)} \int_{0}^{t} \frac{g^{(k)}(\tau)}{(t-\tau)^{q+1-k}} d \tau, \\
\qquad k-1<q<k,
\end{array}
$$

where ${ }_{0}^{c} D_{t}^{q}$ is the Caputo operator, $k$ is the first integer which is not less than $q$, and $g^{(k)}(t)$ is the $k$-order derivative in usual sense of $g(t)$.

Next, based on the 3D autonomous continuous chaotic system reported by Zhou and $\mathrm{Ke}$ [6], a fractional-order system is addressed as

$$
\begin{aligned}
& { }_{0}^{c} D_{t}^{q} x_{1}=-x_{1}+0.5 x_{1} x_{3}+x_{2} x_{3}, \\
& { }_{0}^{c} D_{t}^{q} x_{2}=4 x_{2}-1.2 x_{1} x_{3}, \\
& { }_{0}^{c} D_{t}^{q} x_{3}=x_{1} x_{2}-6 x_{3},
\end{aligned}
$$

where fractional order $0<q<1$. Fractional-order system (2) has five equilibrium points. They are $S_{0}=(0,0,0)$, $S_{1}^{+}=(4.4721,-1.5745,1.1736), S_{1}^{-}=(-4.4721,1.5745$, $1.1736), S_{2}^{+}=(4.4721,3.8106,-2.8403)$, and $S_{2}^{-}=(-4.4721$, $-3.8106,-2.8403)$, respectively.

Now, we can obtain the eigenvalues of the five equilibrium points. The eigenvalues of equilibrium point $S_{0}$ are $(-6,-1$, 4). Thus, equilibrium point $S_{0}$ is an unstable saddle point of index one. The eigenvalues of equilibrium points $S_{1}^{+}$and $S_{1}^{-}$are $(-5.3957,1.4912+3.2168 j, 1.4912-3.2168 j)$. Thus, equilibrium points $S_{1}^{+}$and $S_{1}^{-}$are unstable saddle points of index two. The eigenvalues of equilibrium points $S_{2}^{+}$and $S_{2}^{-}$are $(-6.7558,1.1678+4.7892 j, 1.1678-4.7892 j)$. Thus, equilibrium points $S_{2}^{+}$and $S_{2}^{-}$are unstable saddle points of index two.

Tavazoei and Haeri [17] have obtained that a necessary condition for a fractional-order nonlinear system to exist chaotic is

$$
q \geq \frac{2}{\pi}\left|\arctan \frac{\operatorname{Im}(\lambda)}{\operatorname{Re}(\lambda)}\right|,
$$

where $\lambda$ is the eigenvalues of saddle equilibrium point of index two in fractional-order nonlinear system.
Now, we can obtain the necessary condition of the existence chaos in fractional-order system (2). According to (3), we have the following:

$$
\begin{aligned}
q & \geq \frac{2}{\pi}\left|\arctan \frac{\operatorname{Im}(\lambda)}{\operatorname{Re}(\lambda)}\right| \\
& =0.7237 \text { for equilibrium points } S_{1}^{+} \text {and } S_{1}^{-} ; \\
q & \geq \frac{2}{\pi}\left|\arctan \frac{\operatorname{Im}(\lambda)}{\operatorname{Re}(\lambda)}\right| \\
& =0.8477 \text { for equilibrium points } S_{2}^{+} \text {and } S_{2}^{-} .
\end{aligned}
$$

Thus, the necessary condition of existence chaos in fractionalorder system (2) is $q \geq 0.8477$. This result indicates that fractional-order system (2) with the order as low as 2.5431 can exhibit chaotic attractors.

In this paper, the improved version of Adams-BashforthMoulton [18] (denoted by IVABM) numerical algorithm is used to deal with fractional-order system (2). The IVABM numerical algorithm will be introduced next. Now, consider the fractional-order system

$$
\begin{aligned}
& { }_{0}^{c} D_{t}^{q_{1}} x_{1}=g_{1}\left(x_{1}, x_{2}, x_{3}\right), \\
& { }_{0}^{c} D_{t}^{q_{2}} x_{2}=g_{2}\left(x_{1}, x_{2}, x_{3}\right), \\
& { }_{0}^{c} D_{t}^{q_{3}} x_{3}=g_{3}\left(x_{1}, x_{2}, x_{3}\right)
\end{aligned}
$$

with initial condition $\left(x_{1}(0), x_{2}(0), x_{3}(0)\right)$. Let $l=T / N$, and $t_{n}=n l(n=0,1,2, \ldots, N)$. By IVABM numerical algorithm, system (5) can be discretized as follows:

$$
\begin{aligned}
& x_{1}(n+1)=x_{1}(0) \\
& +\frac{h^{q_{1}}}{\Gamma\left(q_{1}+2\right)}\left[g_{1}\left(x_{1}^{p}(n+1), x_{2}^{p}(n+1), x_{3}^{p}(n+1)\right)\right. \\
& \left.+\sum_{j=0}^{n} \alpha_{1, j, n+1} g_{1}\left(x_{1}(j), x_{2}(j), x_{3}(j)\right)\right], \\
& x_{2}(n+1)=x_{2}(0) \\
& +\frac{h^{q_{2}}}{\Gamma\left(q_{2}+2\right)}\left[g_{2}\left(x_{1}^{p}(n+1), x_{2}^{p}(n+1), x_{3}^{p}(n+1)\right)\right. \\
& \left.+\sum_{j=0}^{n} \alpha_{2, j, n+1} g_{2}\left(x_{1}(j), x_{2}(j), x_{3}(j)\right)\right], \\
& +x_{3}(n+1)=x_{3}(0) \\
& +\frac{h^{q_{3}}}{\Gamma\left(q_{3}+2\right)}\left[g_{3}\left(x_{1}^{p}(n+1), x_{2}^{p}(n+1), x_{3}^{p}(n+1)\right)\right. \\
& \left.\quad+g_{3}\left(x_{1}(j), x_{2}(j), x_{3}(j)\right)\right],
\end{aligned}
$$




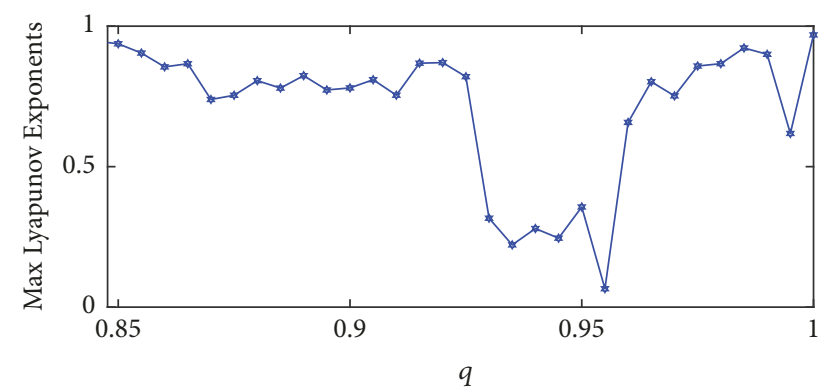

FIGURE 1: The largest Lyapunov exponent varying as fractional order $q$.

where

$$
\begin{aligned}
& x_{1}^{p}(n+1) \\
& =x_{1}(0)+\frac{1}{\Gamma\left(q_{1}\right)} \sum_{j=0}^{n} \beta_{1, j, n+1} g_{1}\left(x_{1}(j), x_{2}(j), x_{3}(j)\right), \\
& x_{2}^{p}(n+1) \\
& =x_{2}(0)+\frac{1}{\Gamma\left(q_{2}\right)} \sum_{j=0}^{n} \beta_{2, j, n+1} g_{2}\left(x_{1}(j), x_{2}(j), x_{3}(j)\right), \\
& x_{3}^{p}(n+1) \\
& =x_{3}(0)+\frac{1}{\Gamma\left(q_{3}\right)} \sum_{j=0}^{n} \beta_{3, j, n+1} g_{3}\left(x_{1}(j), x_{2}(j), x_{3}(j)\right), \\
& \alpha_{i, j, n+1} \\
& =\left\{\begin{array}{ll}
n^{q_{i}+1}-\left(n-q_{i}\right)(n+1)^{q_{i}}, & j=0 \\
(n-j+2)^{q_{i}+1}+(n-j)^{q_{i}+1}-2(n-j+1)^{q_{i}+1}, & 1 \leq j \leq n \\
1, & (i=1,2,3), \\
\beta_{i, j, n+1}=\frac{h^{q_{i}}}{q_{i}}\left[(n-j+1)^{q_{i}}-(n-j)^{q_{i}}\right], &
\end{array},\right. \\
& 0 \leq j \leq n, \quad(i=1,2,3) .
\end{aligned}
$$

The error of this IVABM numerical algorithm is

$$
\begin{aligned}
\left|x_{i}\left(t_{n}\right)-x_{i}(n)\right|=o\left(h^{p_{i}}\right), & \\
& p_{i}=\min \left(2,1+q_{i}\right),(i=1,2,3) .
\end{aligned}
$$

We can yield the largest Lyapunov exponent varying as $q$ via IVABM numerical algorithm, which is shown in Figure 1, where $0.8477 \leq q \leq 1$ and the initial condition is $(-5,2,5)$.

According to Figure 1, we can obtain that the largest Lyapunov exponent is larger zero for $0.8477 \leq q \leq 1$. This result indicates that the chaotic attractor is emerged in fractional-order system (2) for $0.8477 \leq q \leq 1$. For example, let $q=0.8477$, the largest Lyapunov exponent is 0.9853 , and the chaotic attractor in fractional-order system (2) with initial condition $(-5,2,5)$ is shown as Figure 2.

Same as the results reported by Zhou and Ke [6], there are coexisting "positive attractor" and "negative attractor" in fractional-order system (2). The chaotic attractor in fractional-order system (2) can be decided by the initial conditions; that is, the chaotic attractor depends on the distance from the initial point (initial condition $x_{0}=$ $\left(x_{1}(0), x_{2}(0), x_{3}(0)\right)$ to the unstable points [6]. Next, the distances from the initial point to points $S_{1}^{+}, S_{1}^{-}, S_{2}^{+}$, and $S_{2}^{-}$ are denoted by $L_{1}^{+}, L_{1}^{-}, L_{2}^{+}$, and $L_{2}^{-}$, respectively. Same as [6], the following results can be obtained:

(1) If the initial point (initial condition) is near the unstable $S_{1}^{+}$or $S_{2}^{+}$, there is the same chaotic attractor in fractional-order chaotic system (2), which is called "positive attractor" by Zhou and Ke [6], where the "positive attractor" means $x_{1}(t)>0$ and a necessary condition for "positive attractor" is $x_{1}(0)>0$ [6].

(2) If the initial point (initial condition) is near the unstable $S_{1}^{-}$or $S_{2}^{-}$, there is the same chaotic attractor in fractional-order chaotic system (2), which is called "negative attractor" by Zhou and Ke [6], where the "positive attractor" means $x_{1}(t)<0$ and a necessary condition for "negative attractor" is $x_{1}(0)<0$ [6].

Next, some numerical simulations are given for $q=0.9$. Here, the largest Lyapunov exponent is 0.763 for $q=0.9$.

Case 1 (the initial point is near unstable saddle point $S_{1}^{+}$or $\left.S_{1}^{-}\right)$. For example, choose the initial conditions as $(4,-1,1)$. Therefore, $L_{1}^{+}, L_{1}^{-}, L_{2}^{+}$, and $L_{2}^{-}$are $0.7636,8.8563,6.1735$, and 9.7172, respectively. Therefore, the initial point $(4,-1,1)$ is near unstable saddle point $S_{1}^{+}$. Therefore, the fractional-order system (2) has the "positive attractor," which is shown as Figure 3.

For example, choose the initial conditions as $(-4,1,1)$. Therefore, $L_{1}^{+}, L_{1}^{-}, L_{2}^{+}$, and $L_{2}^{-}$are $8.8563,0.7636,9.7172$, and 6.1735 , respectively. Therefore, the initial point $(-4,1,1)$ is near unstable saddle point $S_{1}^{-}$. Thus, fractional-order system (2) has the "negative attractor," which is shown as Figure 3.

Case 2 (the initial point is near unstable saddle point $S_{2}^{+}$or $\left.S_{2}^{-}\right)$. For example, choose the initial conditions as $(4,3,-2)$. Therefore, $L_{1}^{+}, L_{1}^{-}, L_{2}^{+}$, and $L_{2}^{-}$are $5.5875,9.1586,1.2594$, and 10.9026 , respectively. Therefore, the initial point $(4,3,-2)$ is close to unstable saddle point $S_{2}^{+}$. Thus, fractional-order system (2) has the "positive attractor," which is shown as Figure 4.

For example, choose the initial conditions as $(-4,-3,-2)$. Therefore, $L_{1}^{+}, L_{1}^{-}, L_{2}^{+}$, and $L_{2}^{-}$are $9.1586,5.5875,10.9026$, and 1.2594 , respectively. Therefore, the initial point $(-4,-3,-2)$ is close to unstable saddle point $S_{2}^{-}$. Thus, fractional-order system (2) has the "negative attractor," which is shown as Figure 4.

According to Figures 3 and 4, the coexisting chaotic attractors are found in fractional-order chaotic system (2). These results indicate that the chaotic system reported by Zhou and Ke [6] can be extended to its fractional-order version where the coexisting "positive attractor" and "negative attractor" can be observed.

Remark 1. There are overlaps between the coexisting chaotic attractors in [3-5, 7-9]. However, there are two isolated chaotic attractors in fractional-order chaotic system 


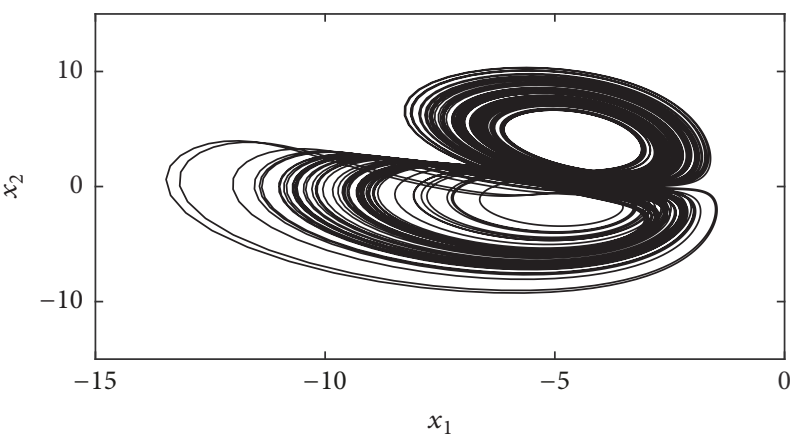

(a)

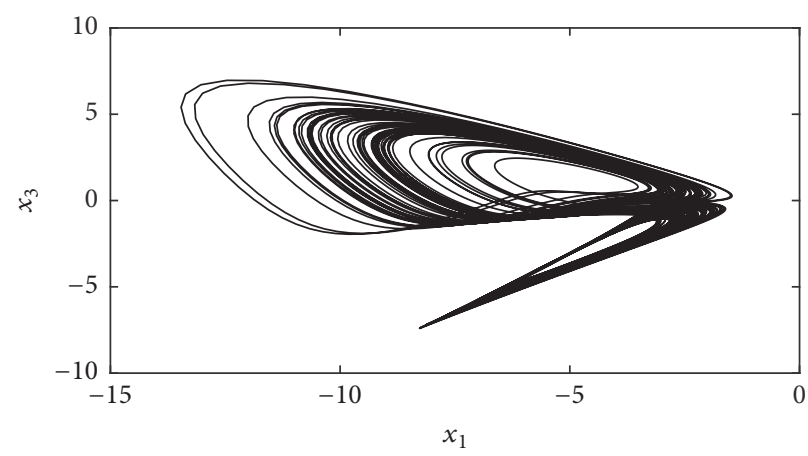

(b)

Figure 2: The chaotic attractor in fractional-order system (2).

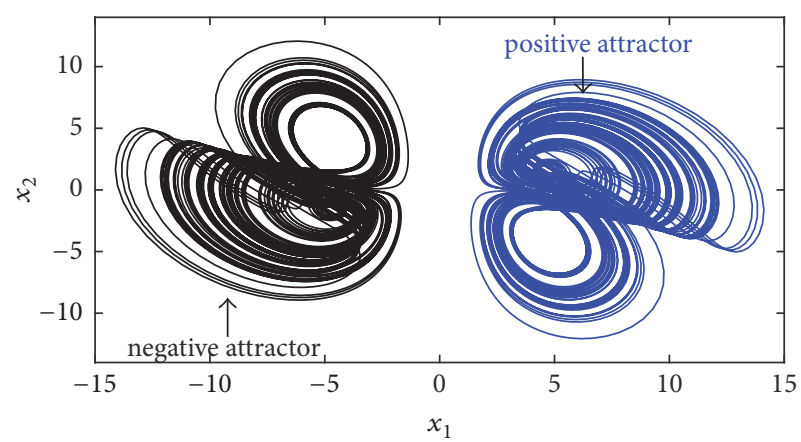

(a)

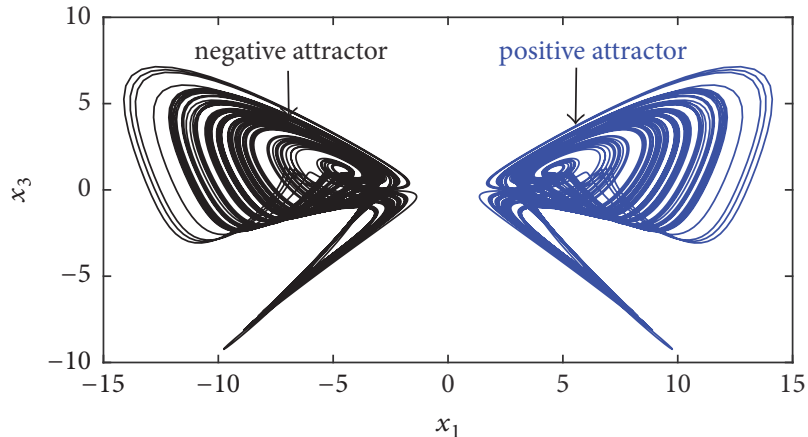

(b)

Figure 3: The "positive attractor" and "negative attractor" in fractional-order system (2).

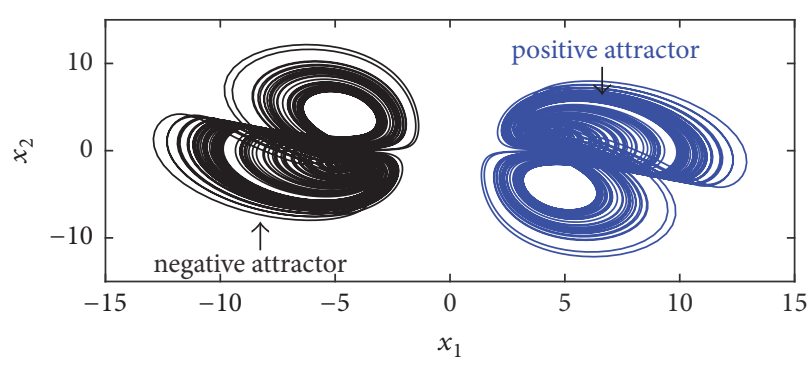

(a)

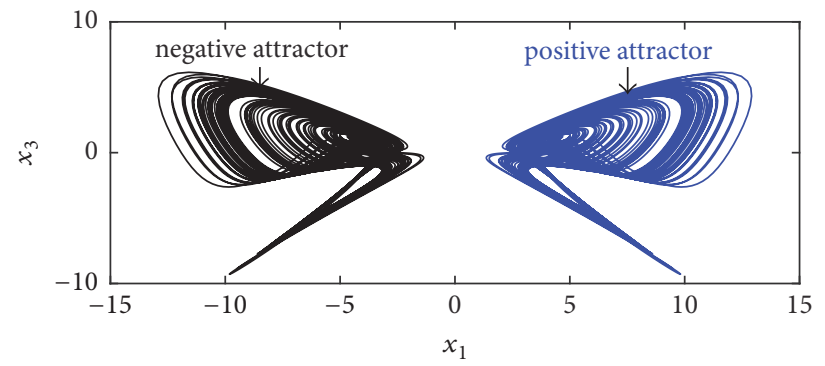

(b)

Figure 4: The "positive attractor" and "negative attractor" in fractional-order system (2).

(2); that is, there are no overlaps between the coexisting chaotic attractors in fractional-order chaotic system (2).

\section{Stabilization of the Unstable Equilibrium Points of the Fractional-Order Chaotic System (2)}

First, a result on stability of fractional-order nonlinear system is recalled. Consider the fractional-order nonlinear system as follows:

$$
{ }_{0}^{c} D_{t}^{q} y(t)=H(y(t))=M y(t)+f(y(t)),
$$

where $0<q \leq 1, y(t)=\left(y_{1}(t), y_{2}(t), \ldots, y_{n}(t)\right)^{T} \in R^{n}$ denotes the state vector, $H: R^{n} \rightarrow R^{n}$ is a nonlinear vector field, $M \in R^{n \times n}$ is a constant matrix, and $f(y(t))$ and $M y(t)$ denote the nonlinear and linear parts of $H(y(t))$.

Lemma 2 (for more details, see [19]). Fractional-order nonlinear system (9) is said to be asymptotically stable if the next two conditions hold:

(i) $\left.f(y(t))\right|_{y(t)=0}=0$, and $\lim _{y(t) \rightarrow 0}(\|f(y(t))\| /\|y(t)\|)=$ 0 ,

(ii) $\left|\arg \lambda_{i}(M)\right|>0.5 q \pi(i=1,2, \ldots, n)$, and $q\|M\|>1$, where $\lambda_{i}(M)$ and $\|M\|$ denote the eigenvalues and the $l_{2}$-norm with respect to matrix $M$, respectively. 
Next, we discuss how to stable the unstable equilibrium point in fractional-order chaotic system (2) via single state variable linear controller. Now, let the unstable equilibrium points in system (2) be $\left(\bar{x}_{1}, \bar{x}_{2}, \bar{x}_{3}\right)$, and the controlled fractional-order system is shown as follows:

$$
\begin{aligned}
& { }_{0}^{c} D_{t}^{q} x_{1}=-x_{1}+0.5 x_{1} x_{3}+x_{2} x_{3}+k_{1}\left(x_{2}-\bar{x}_{2}\right), \\
& { }_{0}^{c} D_{t}^{q} x_{2}=4 x_{2}-1.2 x_{1} x_{3}+k_{2}\left(x_{2}-\bar{x}_{2}\right), \\
& { }_{0}^{c} D_{t}^{q} x_{3}=x_{1} x_{2}-6 x_{3}+k_{3}\left(x_{2}-\bar{x}_{2}\right),
\end{aligned}
$$

where $0.8477 \leq q \leq 1$ and $k_{i}\left(x_{2}-\bar{x}_{2}\right)(i=1,2,3)$ is a linear controller determined by a single state variable $x_{2}$. $k_{i}(i=1,2,3) \in R$ are feedback gains determined later.

Theorem 3. Let real matrix be

$$
A=\left(\begin{array}{ccc}
-\left(1-0.5 \bar{x}_{3}\right) & \left(\bar{x}_{3}+k_{1}\right) & \left(0.5 \bar{x}_{1}+\bar{x}_{2}\right) \\
-1.2 \bar{x}_{3} & \left(4+k_{2}\right) & 1.2 \bar{x}_{1} \\
\bar{x}_{2} & \left(\bar{x}_{1}+k_{3}\right) & -6
\end{array}\right),
$$

and choose suit feedback gains $k_{i}(i=1,2,3)$; if $\left|\arg \lambda_{i}(A)\right|>$ $0.5 q \pi(i=1,2,3)$, then the unstable equilibrium points $\left(\bar{x}_{1}, \bar{x}_{2}, \bar{x}_{3}\right)$ of fractional-order chaotic system (2) in controlled system (10) are asymptotically stable.

Proof. Let $y_{1}=x_{1}-\bar{x}_{1}, y_{2}=x_{2}-\bar{x}_{2}$, and $y_{3}=x_{3}-\bar{x}_{3}$; then controlled system (10) can be changed as

$$
\begin{aligned}
{ }_{0}^{c} D_{t}^{q} y_{1}= & -\left(1-0.5 \bar{x}_{3}\right) y_{1}+\left(\bar{x}_{3}+k_{1}\right) y_{2} \\
& +\left(0.5 \bar{x}_{1}+\bar{x}_{2}\right) y_{3}+0.5 y_{1} y_{3}+y_{2} y_{3}, \\
{ }_{0}^{c} D_{t}^{q} y_{2}= & -1.2 \bar{x}_{3} y_{1}+\left(4+k_{2}\right) y_{2}-1.2 \bar{x}_{1} y_{3}-1.2 y_{1} y_{3}, \\
{ }_{0}^{c} D_{t}^{q} y_{3}= & \bar{x}_{2} y_{1}+\left(\bar{x}_{1}+k_{3}\right) y_{2}-6 y_{3}+y_{1} y_{2} .
\end{aligned}
$$

Thus, the unstable equilibrium points $\left(\bar{x}_{1}, \bar{x}_{2}, \bar{x}_{3}\right)$ in fractional-order chaotic system (2) are changed as the point $\left(y_{1}, y_{2}, y_{3}\right)=(0,0,0)$ in system $(12)$.

One can easily obtain that point $\left(y_{1}, y_{2}, y_{3}\right)=(0,0,0)$ is the origin of system (12). Therefore, the problem of stabilization of the unstable equilibrium points in fractionalorder chaotic system (2) is turned to the problem of stabilization of fractional-order system (12). If the origin of system (12) is asymptotically stable, then the unstable equilibrium points $\left(\bar{x}_{1}, \bar{x}_{2}, \bar{x}_{3}\right)$ of fractional-order chaotic system (2) in controlled system (10) are asymptotically stable.

Now, controlled system (12) can be rewritten as

$$
{ }_{0}^{c} D_{t}^{q} y(t)=A y(t)+f(y(t)),
$$

where $f(y(t))=\left(\left(0.5 y_{1}+y_{2}\right) y_{3}-1.2 y_{1} y_{3} y_{1} y_{2}\right)^{T}$.

First, it is easy to obtain that $\left.f(y(t))\right|_{y(t)=0}=0$, and

$$
\begin{aligned}
& \frac{\|f(y(t))\|}{\|y(t)\|} \\
& \quad=\sqrt{\frac{\left[\left(0.5 y_{1}+y_{2}\right) y_{3}\right]^{2}+\left(-1.2 y_{1} y_{3}\right)^{2}+\left(y_{1} y_{2}\right)^{2}}{y_{1}^{2}+y_{2}^{2}+y_{3}^{2}}}
\end{aligned}
$$

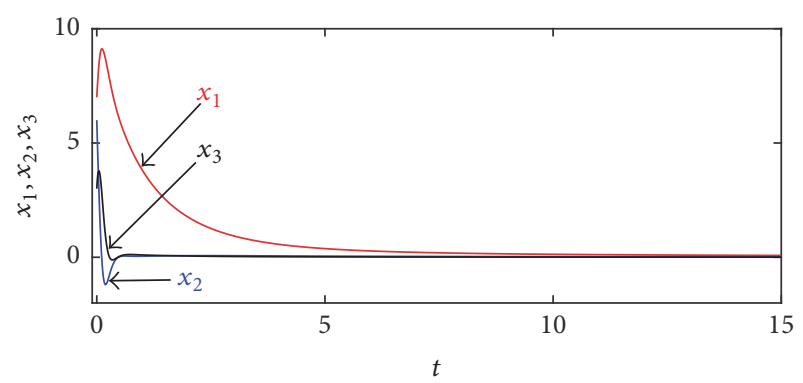

FIgURE 5: The time evolution of state variables $x_{i}(i=1,2,3)$.

$$
\begin{aligned}
& \leq \sqrt{\left[\left(0.5 y_{1}+y_{2}\right)\right]^{2}+\left(-1.2 y_{3}\right)^{2}+\left(y_{2}\right)^{2}}, \\
\lim _{y(t) \rightarrow 0} \frac{\|f(y(t))\|}{\|y(t)\|} & \\
& \leq \lim _{y(t) \rightarrow 0} \sqrt{\left[\left(0.5 y_{1}+y_{2}\right)\right]^{2}+\left(-1.2 y_{3}\right)^{2}+\left(y_{2}\right)^{2}}=0 .
\end{aligned}
$$

Thus, the first condition in Lemma 2 is satisfied.

Second, according to

$$
A=\left(\begin{array}{ccc}
-\left(1-0.5 \bar{x}_{3}\right) & \left(\bar{x}_{3}+k_{1}\right) & \left(0.5 \bar{x}_{1}+\bar{x}_{2}\right) \\
-1.2 \bar{x}_{3} & \left(4+k_{2}\right) & 1.2 \bar{x}_{1} \\
\bar{x}_{2} & \left(\bar{x}_{1}+k_{3}\right) & -6
\end{array}\right),
$$

$q\|A\|>1$, and using $\left|\arg \lambda_{i}(A)\right|>0.5 q \pi(i=1,2,3)$, we can yield that the second condition in Lemma 2 is satisfied.

Therefore, according to Lemma 2, the origin of system (12) is asymptotically stable. That is, the unstable equilibrium points $\left(\bar{x}_{1}, \bar{x}_{2}, \bar{x}_{3}\right)$ of fractional-order chaotic system (2) in controlled system (10) are asymptotically stable. The proof is finished.

Remark 4. In our control scheme, the linear controller is only determined by one single state variable, so our control scheme is different from many previous control schemes.

\section{Numerical Simulations Results}

Next, in order to show the effectiveness of the proposed control approach, the numerical simulations are performed for $q=0.9$.

For the unstable equilibrium point $S_{0}=(0,0,0)$, if we choose $k_{1}=k_{3}=0, k_{2}=-7$, then $A=\left(\begin{array}{ccc}-1 & 0 & 0 \\ 0 & -3 & 0 \\ 0 & 0 & -6\end{array}\right)$. Thus, we obtain $\lambda_{1}=-1, \lambda_{2}=-3, \lambda_{3}=-6$, and $q\|A\|>1$. According to Theorem 3 , the unstable equilibrium point $S_{0}$ in controlled system (10) is asymptotically stable. Figure 5 displays the time evolution of state variables. Here, the initial conditions be $(7,6,3)$.

For the unstable equilibrium point $S_{1}^{+}=(4.4721$, $-1.5745,1.1736)$, if we choose $k_{1}=k_{3}=0, k_{2}=-8$, then $A=\left(\begin{array}{ccc}-0.4132 & 1.1736 & 0.66155 \\ -1.40832 & -4 & 5.36652 \\ -1.5745 & 4.4721 & -6\end{array}\right)$. Thus, we obtain $\lambda_{1}=$ $-0.5726, \lambda_{2,3}=-4.9203 \pm 4.9982 j$, and $q\|A\|>1$. According 


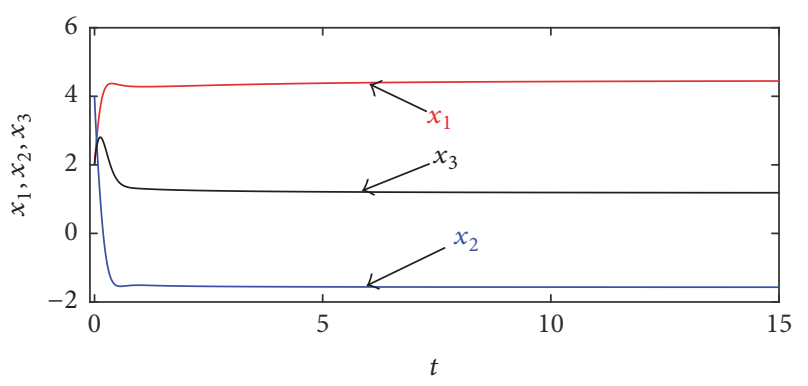

FIgURE 6: The time evolution of state variables $x_{i}(i=1,2,3)$.

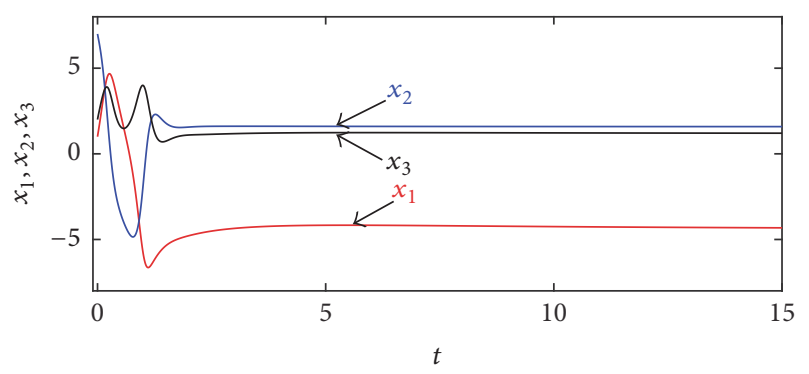

FIGURE 7: The time evolution of state variables $x_{i}(i=1,2,3)$.

to Theorem 3, the unstable equilibrium point $S_{1}^{+}$in controlled system (10) is asymptotically stable. Figure 6 shows the time evolution of state variables. Here, the initial conditions are $(2,4,2)$.

For the unstable equilibrium point $S_{1}^{-}=(-4.4721$, $1.5745,1.1736)$, if we choose $k_{1}=k_{3}=0, k_{2}=-4$, then $A=$ $\left(\begin{array}{ccc}-0.4132 & 1.1736 & -0.66155 \\ -1.40832 & 0 & -5.36652 \\ 1.5745 & -4.4721 & -6\end{array}\right)$ Therefore, we obtain $\lambda_{1}=-0.5418$, $\lambda_{2,3}=-2.9357 \pm 4.1682 j$, and $q\|A\|>1$. According to Theorem 3 , the unstable equilibrium point $S_{1}^{-}$in controlled system (10) is asymptotically stable. Figure 7 shows the time evolution of state variables. Here, the initial conditions are $(1,7,2)$.

For the unstable equilibrium point $S_{2}^{+}=(4.4721,3.8106$, $-2.8403)$, if we choose $k_{1}=-2.1597, k_{2}=-7, k_{3}=$ -9.4721, then $A=\left(\begin{array}{ccc}-2.42015 & -5 & 6.04665 \\ 3.40836 & -3 & 5.36652 \\ 3.8106 & -5 & -6\end{array}\right)$. Thus, we obtain $\lambda_{1}=$ $-10.8877, \lambda_{2,3}=-0.2662 \pm 1.040 j$, and $q\|A\|>1$. According to Theorem 3 , the unstable equilibrium point $S_{2}^{+}$in controlled system (10) is asymptotically stable. Figure 8 shows the time evolution of state variables. Here, the initial conditions are $(5,4,-3)$.

For the unstable equilibrium point $S_{2}^{-}=(-4.4721$, $-3.8106,-2.8403)$, if we choose $k_{1}=-7.1597, k_{2}=-7, k_{3}=$ 9.9721, then $A=\left(\begin{array}{ccc}-2.42015 & -10 & -6.04665 \\ 3.40836 & -3 & -5.36652 \\ -3.8106 & 5.5 & -6\end{array}\right)$. Thus, we obtain $\lambda_{1}=$ $-9.3229, \lambda_{2,3}=-1.0486 \pm 0.8095 j$, and $q\|A\|>1$. According to Theorem 3 , the unstable equilibrium point $S_{2}^{-}$in controlled system (10) is asymptotically stable. Figure 9 shows the time evolution of state variables. Here, the initial conditions are $(-7,-6,-2)$.

The simulative results in Figures 5-9 show the effectiveness of our control scheme.

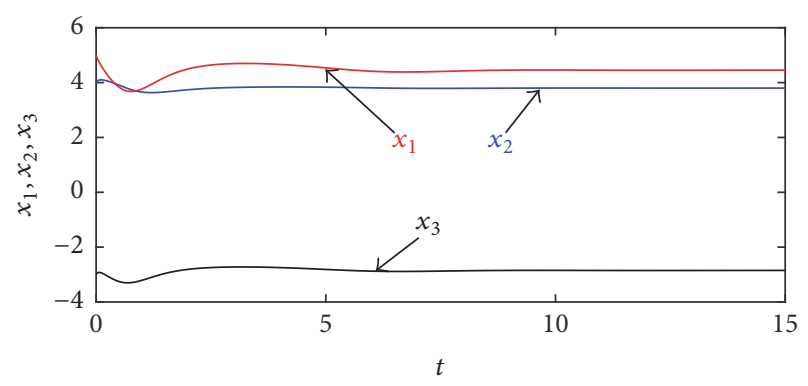

FIgURE 8: The time evolution of state variables $x_{i}(i=1,2,3)$.

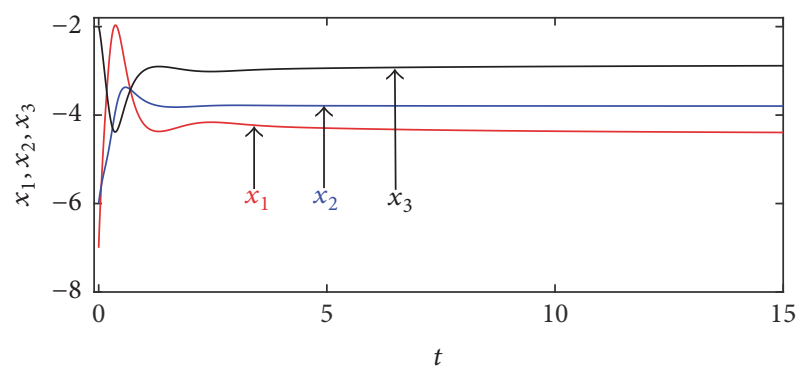

FIGURE 9: The time evolution of state variables $x_{i}(i=1,2,3)$.

\section{Conclusions}

In this paper, a fractional-order chaotic system is proposed. The necessary condition of existence chaos in this fractionalorder chaotic system is $q \geq 0.8477$. The largest Lyapunov exponent varying as fractional order $q$ is given. The coexisting "positive attractor" and "negative attractor" can be observed, and the necessary conditions for "positive attractor" and "negative attractor" are obtained. Meanwhile, by a single state variable, a linear controller is used for the stabilization of the unstable equilibrium points of the fractional-order chaotic system. The numerical results show that the control approach is effective.

\section{Data Availability}

The data used to support the findings of this study are available from the corresponding author upon request.

\section{Conflicts of Interest}

The authors declare no conflicts of interest.

\section{References}

[1] G. R. Chen and X. Dong, From Chaos to Order Perspectives, Methodologies and Applications, World scientific, Singapore, 1998.

[2] J. C. Sprott, Chaos and Time-Series Analysis, Oxford University Press, Oxford, UK, 2003.

[3] V.-T. Pham, C. Volos, S. Jafari, and T. Kapitaniak, "Coexistence of hidden chaotic attractors in a novel no-equilibrium system," Nonlinear Dynamics, vol. 87, no. 3, pp. 2001-2010, 2017. 
[4] B. Bao, H. Qian, J. Wang et al., "Numerical analyses and experimental validations of coexisting multiple attractors in Hopfield neural network," Nonlinear Dynamics, vol. 90, no. 4, pp. 2359-2369, 2017.

[5] X. Wang, S. Vaidyanathan, C. Volos, V.-T. Pham, and T. Kapitaniak, "Dynamics, circuit realization, control and synchronization of a hyperchaotic hyperjerk system with coexisting attractors," Nonlinear Dynamics, vol. 89, no. 3, pp. 1673-1687, 2017.

[6] P. Zhou and M. Ke, "A new 3d autonomous continuous system with two isolated chaotic attractors and its topological horseshoes," Complexity, vol. 2017, Article ID 4037682, 7 pages, 2017.

[7] F. Yuan, G. Y. Wang, and Y. R. Shen, "Coexisting attractors in a memcapacitor-based chaotic oscillator," Nonlinear Dynamics, vol. 85, no. 1, pp. 37-50, 2016.

[8] Q. Lai and S. Chen, "Coexisting attractors generated from a new $4 \mathrm{D}$ smooth chaotic system," International Journal of Control, Automation, and Systems, vol. 14, no. 4, pp. 1124-1131, 2016.

[9] J. Kengne, Z. T. Njitacke, and H. B. Fotsin, "Dynamical analysis of a simple autonomous jerk system with multiple attractors," Nonlinear Dynamics, vol. 83, no. 1-2, pp. 751-765, 2016.

[10] R. Hilfer, Applications of Fractional Calculus in Physics, World Scientific, Singapore, 2000.

[11] I. Podlubny, Fractional Differential Equations, Academic Press, San Diego, Calif, USA, 1999.

[12] P. Zhou and P. Zhu, "A practical synchronization approach for fractional-order chaotic systems," Nonlinear Dynamics, vol. 89, no. 3, pp. 1719-1726, 2017.

[13] P. Zhou, R. J. Bai, and J. M. Zheng, "Stabilization of a fractionalorder chaotic brushless DC motor via a single input," Nonlinear Dynamics, vol. 82, no. 1-2, pp. 519-525, 2015.

[14] T. T. Hartley, C. F. Lorenzo, and H. K. Qammer, "Chaos in a fractional order Chua's system," IEEE Transactions on Circuits and Systems I: Fundamental Theory and Applications, vol. 42, no. 8, pp. 485-490, 1995.

[15] M. P. Aghababa, "Chaos in a fractional-order micro-electromechanical resonator and its suppression," Chinese Physics $B$, vol. 21, no. 10, Article ID 100505, 9 pages, 2012.

[16] M. P. Aghababa and H. P. Aghababa, "The rich dynamics of fractional-order gyros applying a fractional controller," Proceedings of the Institution of Mechanical Engineers, Part I: Journal of Systems and Control Engineering, vol. 227, no. 7, pp. 588-601, 2013.

[17] M. S. Tavazoei and M. Haeri, "A necessary condition for double scroll attractor existence in fractional-order systems," Physics Letters A, vol. 367, no. 1-2, pp. 102-113, 2007.

[18] C. Li and G. Peng, "Chaos in Chen's system with a fractional order," Chaos, Solitons \& Fractals, vol. 22, no. 2, pp. 443-450, 2004.

[19] L. Chen, Y. Chai, R. Wu, and J. Yang, "Stability and stabilization of a class of nonlinear fractional-order systems with caputo derivative," IEEE Transactions on Circuits and Systems II: Express Briefs, vol. 59, no. 9, pp. 602-606, 2012. 


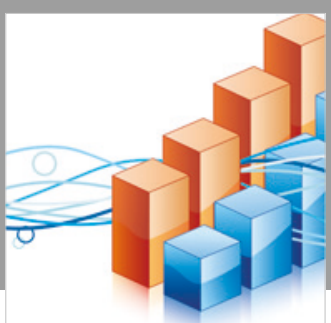

Advances in

Operations Research

\section{-n-m}
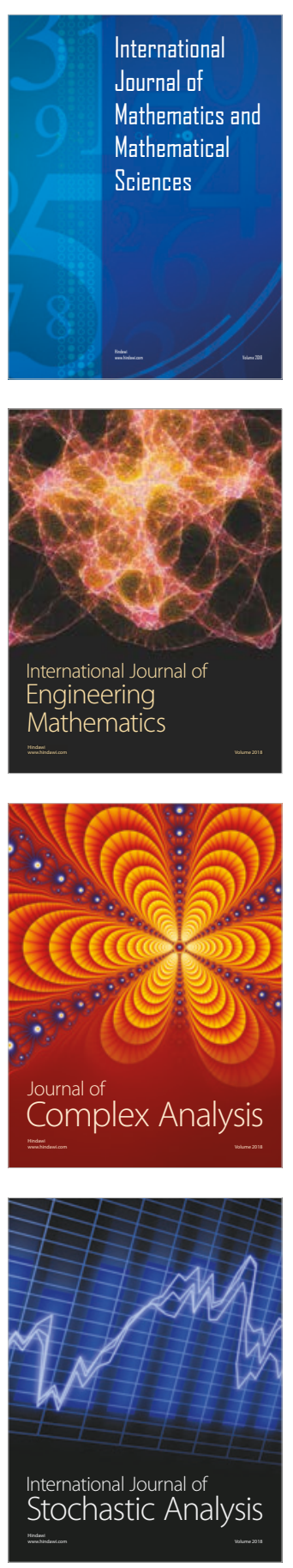
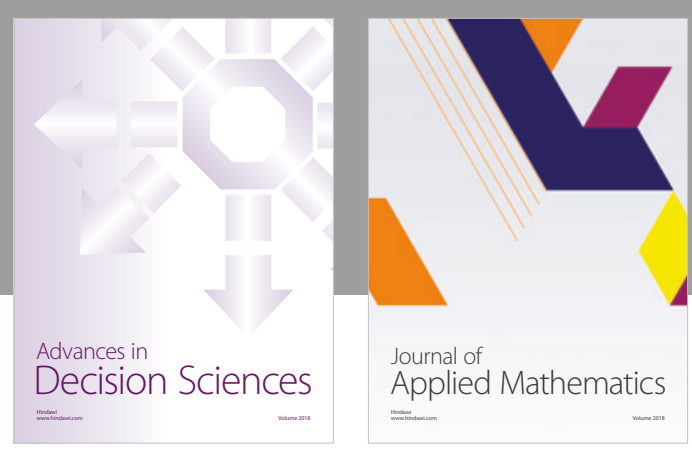

Journal of

Applied Mathematics
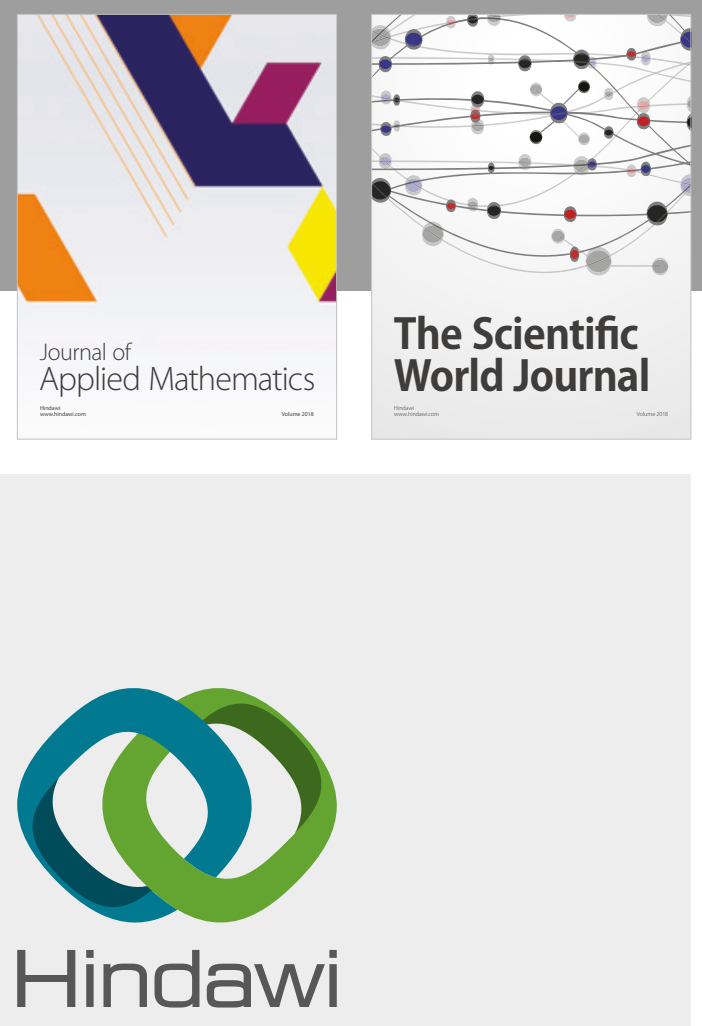

Submit your manuscripts at

www.hindawi.com

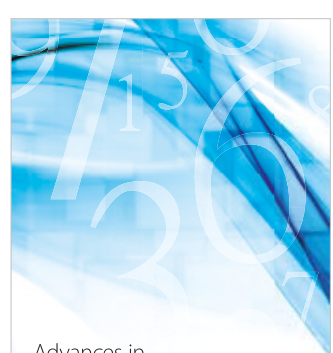

Advances in
Numerical Analysis
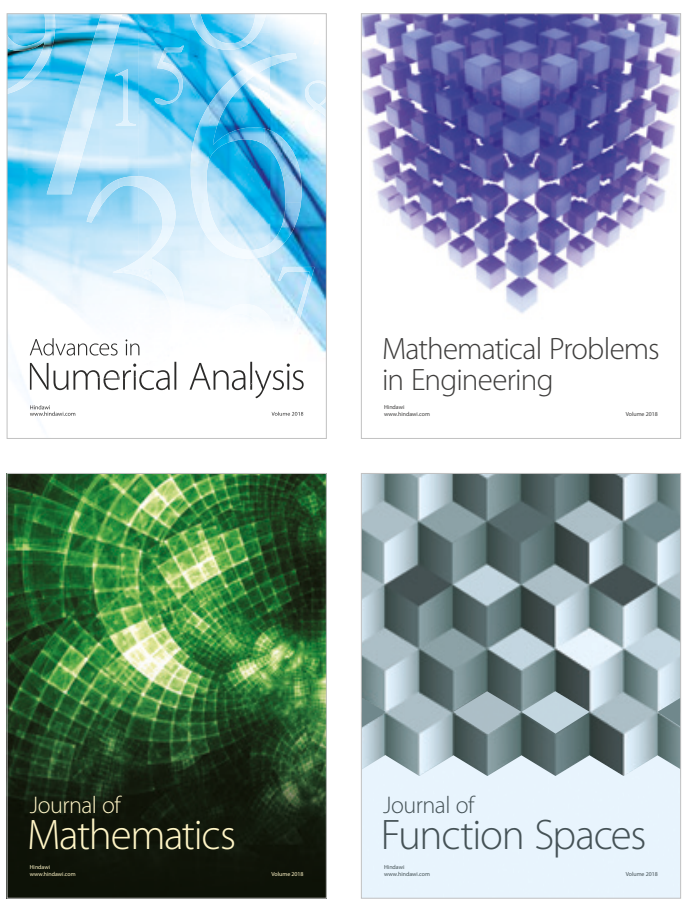

Mathematical Problems in Engineering

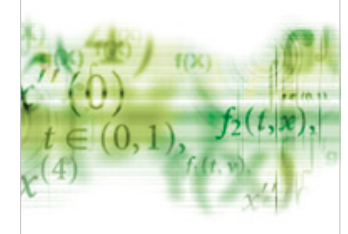

International Journal of

Differential Equations

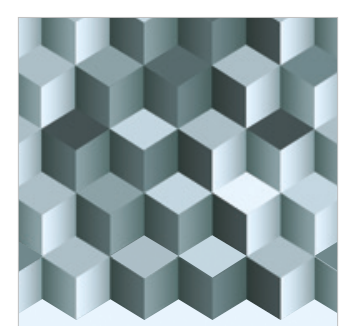

Journal of

Function Spaces
The Scientific

World Journal

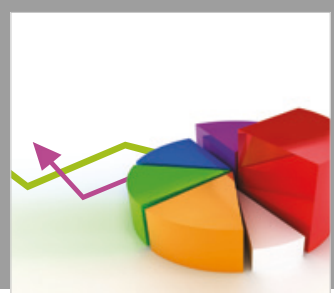

Journal of

Probability and Statistics
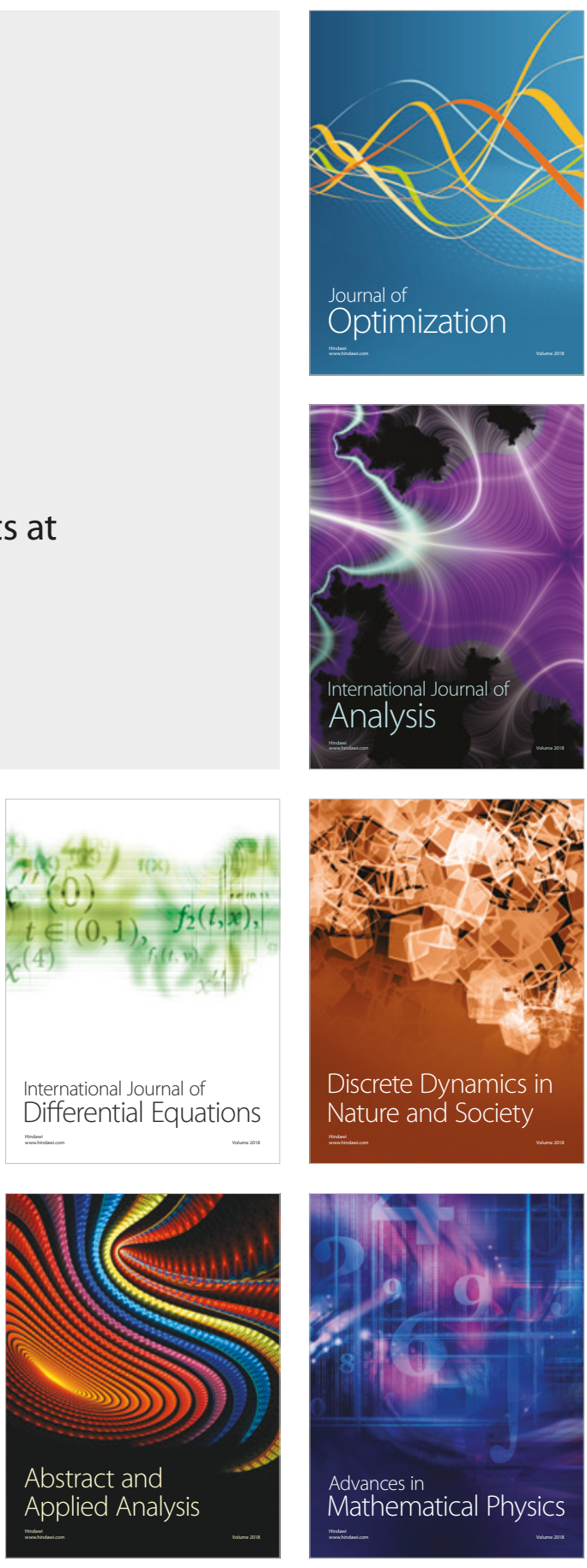